\title{
Intraoperative Ear Injury
}

National Cancer Institute

\section{Source}

National Cancer Institute. Intraoperative Ear Injury. NCI Thesaurus. Code C78378.

Damage to the ear during a surgical procedure. 